\title{
Frontières
}

\section{Le syndrome de Diogène}

\section{À la recherche de l'esprit au-delà de la souillure}

\section{Jean-Jacques Naef}

Volume 13, numéro 2, printemps 2001

Les morts de l'esprit

URI : https://id.erudit.org/iderudit/1074458ar

DOI : https://doi.org/10.7202/1074458ar

Aller au sommaire du numéro

\section{Éditeur(s)}

Université du Québec à Montréal

ISSN

1180-3479 (imprimé)

1916-0976 (numérique)

Découvrir la revue

Citer cet article

Naef, J.-J. (2001). Le syndrome de Diogène : à la recherche de l'esprit au-delà de la souillure. Frontières, 13(2), 54-57. https://doi.org/10.7202/1074458ar
Résumé de l'article

Le "syndrome de Diogène ", entité médico-psychosociale, définie en Angleterre il y a trente ans, nous interpelle en tant que soignant dans la communauté. La prise en charge de ces patients dans le refus conduisait à des intervention en urgence, alors que leur état de santé s'était dégradé à un point tel que les deux tiers devaient décéder lors de leur admission en milieu hospitalier. Ces patients dans le refus, l'isolement et la crasse, vivant comme des " clochards " à domicile nous préoccupent. Que faire pour diminuer cette mortalité catastrophique ? Ces personnes avaient-elles leur capacité de discernement ? Leur esprit n'était-il pas suffisamment perturbé pour que nous puissions nous passer de leur avis pour intervenir sans leur consentement ? À l'aide de quelques situations, nous essaierons de montrer que l'esprit de ces personnes est bien vivant et qu'il est possible de maintenir ces personnes à domicile et de diminuer de façon radicale les taux de mortalité observés.
Ce document est protégé par la loi sur le droit d'auteur. L'utilisation des services d'Érudit (y compris la reproduction) est assujettie à sa politique d'utilisation que vous pouvez consulter en ligne.

https://apropos.erudit.org/fr/usagers/politique-dutilisation/ 
Résumé

Le "syndrome de Diogène", entité médico-psychosociale, définie en Angleterre il y a trente ans, nous interpelle en tant que soignant dans la communauté. La prise en charge de ces patients dans le refus conduisait à des intervention en urgence, alors que leur état de santé s'était dégradé à un point tel que les deux tiers devaient décéder lors de leur admission en milieu hospitalier. Ces patients dans le refus, l'isolement et la crasse, vivant comme des "clochards » à domicile nous préoccupent. Que faire pour diminuer cette mortalité catastrophique? Ces personnes avaient-elles leur capacité de discernement? Leur esprit n'était-il pas suffisamment perturbé pour que nous puissions nous passer de leur avis pour intervenir sans leur consentement? À l'aide de quelques situations, nous essaierons de montrer que l'esprit de ces personnes est bien vivant et qu'il est possible de maintenir ces personnes à domicile et de diminuer de façon radicale les taux de mortalité observés.

Mots clés: syndrome de Diogène misère sociale - isolement social-droits du patient

\section{Abstract \\ Diogene's Syndrome, a medico-psycho- social entity first defined in England 30 years ago, is a challenging issue for care-givers in the community. Manage- ment of these patients who refuse any help has led to emergency interventions, when their state of health was so damaged that two-thirds were to die during their hospitalizations. These patients in deny, isolation and filth, living as "tramps" in their own home is a real issue. What could be done to diminish this catastrophic mortality? Have these people their full capacity of judgement? Aren't their minds sufficiently disturbed that we could justifiably intervene without their full consent? Based on a few situations, we will try to demonstrate that the mind of these people is well alive, and that it is possible to maintain them in their homes and to drastically diminish the observed mortality rates.}

Key words: Diogene's Syndrome - social breakdown - isolation - patient's rights

\section{Le syndrome de Diogène À la recherche de l'esprit au-delà de la souillure}

Jean-Jacques Naef,

médecin, Policlinique de gériatrie

et Département de médecine communautaire,

Hôpitaux universitaires de Genève; détenteur du Certificat de formation continue en éthique clinique, Centre interfacultaire de gérontologie

et Faculté de médecine, Université de Genève, Suisse.

\section{LA PROBLÉMATIQUE DU SYNDROME DE DIOGÈNE}

HISTORIQUE

Diogène le Cynique, philosophe grec né à Sinope au $3^{e}$ siècle avant J.-C., méprisait les conventions sociales et refusait de se laisser enfermer dans de telles règles. L'histoire raconte qu'il vivait dans un tonneau. Elle dit aussi qu'on le rencontra un jour dans les rues d'Athènes, en plein midi, une lanterne allumée à la main, déclarant: "Je cherche un homme. »

En 1966, MacMillan et Shaw publient dans le British Medical Journal une étude sur des personnes âgées ne répondant pas aux standards d'hygiène et d'environnement, pouvant répondre aux critères d'un syndrome ${ }^{1}$. Ils parlent de déstructuration des phases de la sénilité (senile breakdown). L'image la plus fréquemment citée de ces personnes est celle d'une vieille femme vivant seule, occasionnellement des hommes ou des couples, dont l'aspect physique et environnementalest repoussant. Ces personnes sont pouilleuses, des selles et de l'urine maculent l'appartement.
Le degré de tolérance du voisinage à l'égard de ces situations est souvent étonnamment élevé. En fin de compte, la police ou les services équivalant à nos services d'hygiène sont appelés. Ces services officiels sont souvent démunis et l'on fait appel à de multiples organismes pour essayer de résoudre ces situations, généralement avec peu de succès.

\section{DÉFINITION ET PROBLÉMATIQUE}

En 1975, Clark, Mankikar et Gray définissent pour la première fois «le syndrome de Diogène $»^{2}$ dans le cadre d'une étude clinique au Brighton General Hospital. Les critères retenus pour définir ce syndrome sont les suivants :

- appartement sale et en désordre,

- aspect personnel repoussant,

- amoncellement de déchets

(syllogomanie) occasionnellement,

- absence de honte ou de culpabilité relative à leur situation,

- refus de l'aide offerte,

- situation financière dans les normes,

- personnalité particulière.

Cette dernière étude mettait en évidence un taux de mortalité extrêmement important dans les cas de prise en charge hospitalière, soit de décès en milieu psychiatrique (80,95\% de décès en milieu somatique). Par ailleurs, le taux de décès en fin d'étude s'élevait globalement à $41,67 \%$ pour les hommes et à $51,67 \%$ pour les femmes, dont $4,17 \%$ ont été attribués directement au syndrome. 


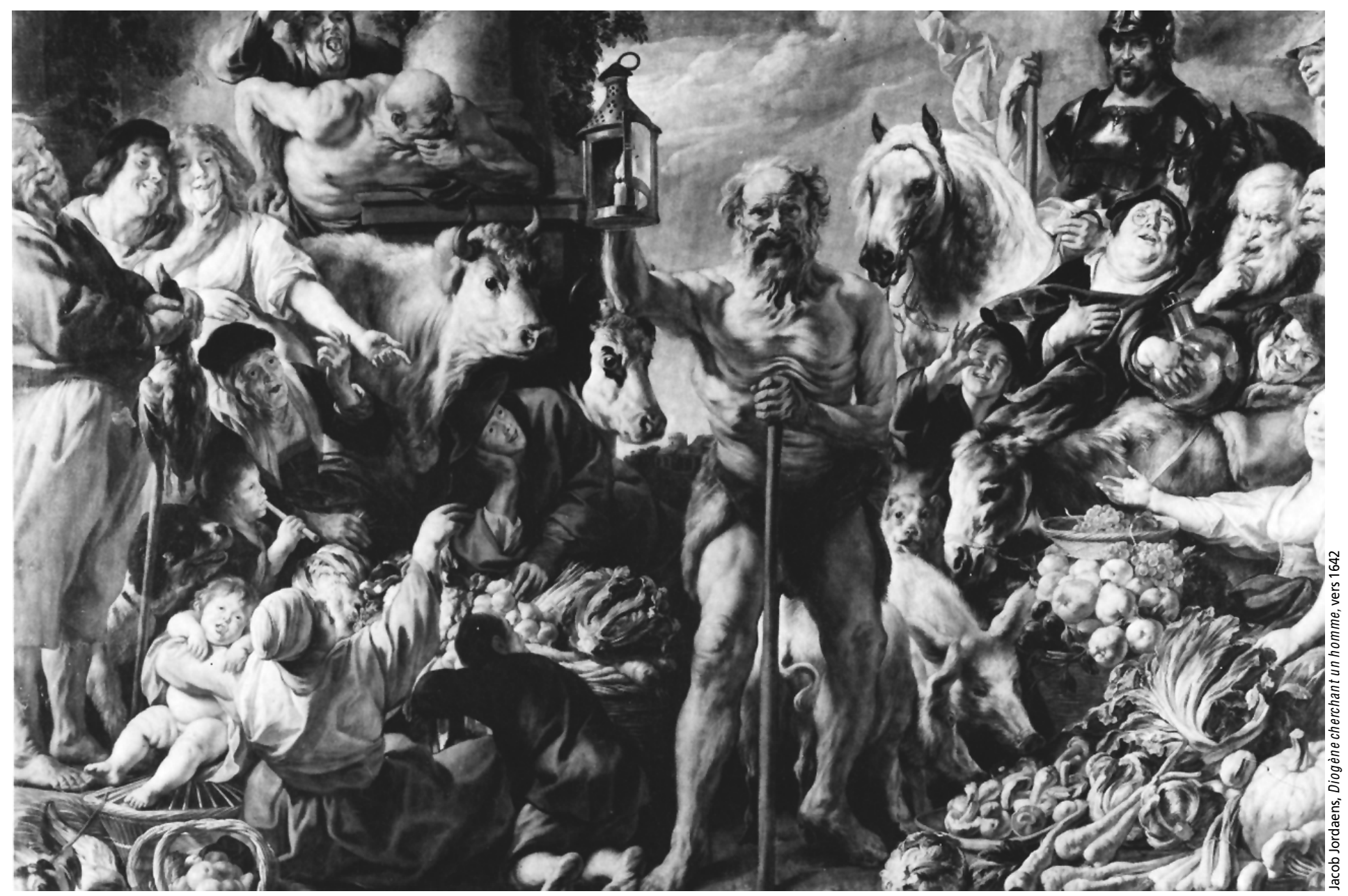

Quelques articles ${ }^{3}$ essentiellement descriptifs de ces situations difficiles ont été publiés à ce jour. On retrouve le même syndrome sous les termes de Social Breakdown in Elderly, Self-neglect in Elderly. Dans le cadre d'un travail de thèse, nous avons cherché à étudier la situation dans le canton de Genève. Nous avons répertorié et analysé 126 situations entre 1980 et 1995.

\section{QUELQUES DONNÉES \\ ÉPIDÉMIOLOGIQUES}

SUR LA PROBLÉMATIQUE À GENÈVE

Dans notre étude, nous avons répertorié les personnes d'âge gériatrique (supérieur à 62 ans pour les femmes et à 65 ans pour les hommes) du canton de Genève, répondant aux critères du syndrome de Diogène, tels que définis par Clark et al. en 1975.

Pour ce faire, nous avons eu accès aux fichiers du Service du médecin cantonal, aux dossiers du Département de gériatrie et en particulier ceux de la Policlinique de gériatrie. Nous avons recensé les cas répondant aux critères de ce syndrome entre janvier 1980 et septembre 1995 dans le canton de Genève.

Ainsi, 126 patients présentant un syndrome de Diogène ont pu être recensés dans le canton de Genève, pendant les 15 années prises en considération (prévalence 0,2\% de la population âgée de plus de 65 ans). Les femmes (âge moyen 81,7 ans) représentent $66,12 \%$ du collectif et les hommes (âge moyen 80,4 ans) $33,88 \%$. Nous avons répertorié cinq couples mariés et deux couples de sœurs. Une patiente vivait avec sa fille et sa petite-fille, ces deux dernières n'entraient pas en considération pour l'étude.

En moyenne, il a fallu 37 semaines pour pouvoir trouver une ébauche de solution, voire une solution définitive; $56 \%$ ont refusé partiellement l'intervention proposée, $40 \%$ des patients ont d'emblée refusé toute intervention et $4 \%$ ont accepté d'emblée une aide. De nombreuses « réinterventions » ont été nécessaires.

\section{SITUATIONS CLINIQUES DE SYNDROME DE DIOGĖNE À GENĖVE} RENATA

Originaire de Pologne, Renata fuit son pays dans les années 1930 bien après la révolution russe d'octobre 1917, alors qu'elle n'a que 16 ans. Venue en Suisse, elle était bien intégrée et a mené une carrière dans les institutions internationales comme secrétaire de direction. Elle a toujours été d'un caractère solitaire, ses relations amicales étaient souvent des contacts de travail mais peu d'amis véritables. À la retraite, elle s'est isolée de plus en plus, effectuant paradoxalement une croisière en mer chaque année durant un mois.

Renata a 76 ans quand elle est signalée par les autorités sanitaires du canton. Ces dernières nous ont mandatés pour évaluer la situation et répondre à une plainte des locataires de son immeuble : mauvaises odeurs émanant du balcon et de la porte d'entrée.

Nous avons écrit à plusieurs reprises à la patiente pour la rencontrer, sans succès. Ce préalable infructueux, nous décidons de nous présenter sans préavis. Le médecin et l'assistante sociale requis en équipe mobile ont d'abord parlementé sur le palier pendant un long moment pour entrer, sans succès. L'odeur était effectivement difficilement supportable. L'équipe est revenue à la charge quelques jours plus tard sans plus de résultats. Après six mois de dialogue sur le palier, Renata a enfin entrouvert sa porte. Le spectacle est hallucinant: couloir encombré de cartons jusqu'au plafond, cages empilées avec animaux vivants en grand nombre. Le reste de l'appartement n'est pas visible, car trop encombré. Elle ne nous laisse toujours pas entrer. Pour ce faire, il faudra encore un mois de travail. 
Dans l'intervalle, nous avons dû négocier avec le voisinage et le service du médecin cantonal pour que Renata ne soit pas expulsée. La présence d'animaux a motivé l'intervention des services compétents, ils ont été évacués immédiatement avec une interdiction de reprendre des animaux à domicile.

Ce moment fut particulièrement difficile pour la patiente et le contact a failli être rompu à nouveau.

Une nouvelle période de négociation a commencé, pour pouvoir procéder au nettoyage de l'appartement, particulièrement à l'évacuation des ordures, qui étaient le nœud gordien du conflit avec le voisinage.

Renata a toujours refusé une évaluation médicale mais elle a accepté des entretiens avec notre assistante sociale qui la rencontrait au café du quartier.

Des animaux se trouvaient une nouvelle fois dans son domicile et ils ont été évacués à nouveau par les autorités sanitaires.

Elle est venue dans nos locaux, bien plus tard et sans préavis pour se plaindre d'une grosseur à la joue. Nous avons mis deux mois pour la convaincre de se laisser examiner. Elle a finalement accepté de voir avec nous les médecins spécialistes qui confirmaient que la situation était dépassée au plan curatif.

Ses forces ont progressivement diminué, et Renata nous a demandé de l'hospitaliser. Elle a été admise dans notre établissement de soins palliatifs où elle décéda quelques semaines plus tard.

\section{MARIE-LOUISE}

Les faits remontaient à 10 ans; ils avaient fait la manchette des journaux : «Deux tonnes d'ordures évacuées d'une mercerie en pleine ville de Genève».

J'avais pris connaissance pour la première fois du cas de Marie-Louise après la lecture d'une fiche signalétique émanant du médecin cantonal, d'un article de presse et d'un rapport de police.

Il y a deux ans, nous avions été sollicités par un établissement pour personnes âgées la concernant. Marie-Louise avait des problèmes de comportement, elle refusait de changer sa vieille jaquette trouée et de mettre des habits neufs que les infirmières lui avaient achetés. Cela dérangeait au plus haut point les résidents, surtout au moment convivial des repas.

Lorsque j'ai pénétré dans la chambre de Marie-Louise, j'ai trouvé une vieille dame de 87 ans, méfiante, peu encline à me parler. J'ai été d'emblée frappé par trois piles de journaux, bien rangées, dans la minuscule chambre. C'est à ce moment que j'ai établi la relation avec la personne signalée 10 ans auparavant.

Nous avons pu nous entretenir sur ce qui lui était arrivé et sur le motif de son refus d'intégration, dans l'établissement où elle avait été admise contre son gré. Des mesures légales restrictives avaient été prises à son encontre (tutelle).

\section{LES DIFFÉRENTES APPROCHES FACE AUX PATIENTS PRÉSENTANT UN SYNDROME DE DIOGĖNE}

À deux problématiques répondent deux approches.

Dans le cas de Renata, une approche « douce », qui permet de respecter son autonomie et sa liberté, dans le cas de MarieLouise, une approche plus juridique, avec à la clé des mesures restrictives qui la privent en partie de ses droits.

Notre objectif dans ces situations est d'établir un pont, une relation de confiance entre le patient et une personne de référence. Cette dernière peut être indistinctement l'un des intervenants de santé soit une assistante sociale, une aide ménagère, une infirmière, un ou une psychologue, voire le médecin lui-même.

Y. Kocher (psychologue) et M.J. Chabert (infirmière en santé publique) ont étudié de façon détaillée 18 situations qui nous ont été référées ${ }^{4}$. Sur le plan de la santé mentale, on relève huit personnes avec un affaiblissement intellectuel compatible avec l'âge, quatre personnes avec une démence moyennement avancée, une personne avec une démence avancée, deux personnes souffrant d'un syndrome amnésique et trois d'un trouble délirant schizoparanoïde (DSMIIIR $^{5}$ ). On retrouve ainsi des personnalités paranoïaques (7), schizoïdes (3), antisociales (2), schizotypiques (3), indéterminées (3).

\section{L'APPROCHE RIGIDE}

Cette étude illustre à notre sens «la capacité de discernement» conservée par ces personnes, peut-être à l'exception des cas de démence avancée. «La marginalité du comportement» des personnes présentant un syndrome de Diogène n'a jamais été reconnue par les psychiatres comme une affection mentale ${ }^{6}$.

Dans ce sens, il est difficile de ne pas respecter l'autonomie de ces patients. En effet, démontrer qu'ils n'ont pas leur capacité de discernement est du ressort médical, en principe sur mandat judiciaire (en Suisse, la Chambre des tutelles). Il s'agit dès lors d'une décision d'expert. En pratique quotidienne, chaque médecin autorisé à pratiquer peut interner un malade (loi genevoise K1.12). Sa décision peut alors être soumise à recours du patient ou de ses proches. Il faut démontrer l'urgence de la situation, la mise en danger du patient pour lui-même ou pour autrui et la nécessité d'être traité en milieu spécialisé psychiatrique.

Parfois, la décision est prise par les autorités sanitaires qui estiment que la santé publique est mise en danger. Cette décision est suivie d'une expulsion, d'un nettoyage et d'une réfection du logement.

Nous n'avons pas pu démontrer que cette attitude entraînait un surcroît de mortalité, comme dans les études de McMillan puis de Clark.

Cette pratique a été bannie d'emblée, vu les difficultés à démontrer dans l'urgence l'existence d'un syndrome de Diogène, le patient étant en opposition totale. Ces démarches prennent du temps. Or le temps de latence, d'inertie de la justice ou la durée de tolérance du voisinage incommodé n'ont pas la même valeur que celui qui est nécessaire à la médecine. Autrement dit, « savoir laisser du temps au temps » pour régler de telles situations apparaît médicalement le plus efficace. Faire cohabiter pacifiquement ces deux impératifs est souvent un exercice de funambule difficile à gérer pour le personnel de santé.

\section{L'APPROCHE SENSIBLE}

L'approche «douce», visant à " apprivoiser» le patient est préférée actuellement. La philosophie du service étant de privilégier le maintien à domicile, nous intervenons en équipe multidisciplinaire, en essayant d'introduire un soignant de référence. Comme nous l'évoquions, cette attitude demande beaucoup de persévérance, plusieurs semaines pour pouvoir entrer en contact (15 en moyenne) et encore plus pour pouvoir ébaucher un début de solution.

Cette façon de faire, souvent mal comprise du voisinage, nous oblige à négocier, non seulement avec le patient, mais également avec le voisinage et les autorités.

Les éléments évoqués sont souvent en relation avec la difficulté d'accepter la différence, l'indécence de laisser vivre l'autre avec des standards différents, la crainte d'être envahi ou infecté par la vermine.

La pression des propriétaires de logement est également importante, face à la dégradation potentielle ou réelle des lieux occupés par un patient avec un syndrome de Diogène.

Souvent les mêmes difficultés sont vécues par l'équipe soignante. Elle a l'impression d'être impuissante et le risque d'épuisement est grand. Dans ce sens, un travail de partage et de soutien face aux difficultés rencontrées est indispensable, lors de colloques multidisciplinaires par exemple. C'est une occasion d'élargir son point de vue et d'éviter de s'enfermer dans une vision univoque de la situation qui pourrait amener l'intervenant à agir plus selon ses sentiments personnels que selon une voie raisonnée et surtout concertée.

L'isolement «volontaire» du patient, nous oblige à laisser en marge notre mission première qui est de veiller à son état 
de santé. Il s'agit de consolider la relation, de rechercher un réseau potentiel, un lien qui pourrait nous aider à entrer en relation et nous permettre de rompre cet isolement.

\section{QUELQUES ÉLÉMENTS DE RÉFLEXION SUR LA PRÉVENTION DU SYNDROME DE DIOGĖNE}

En reprenant plusieurs histoires de vie, avec les patients ou leur entourage, nous avons pu mettre en évidence un profil assez semblable au cours de leur vie active. Sans avoir pu réaliser une analyse de personnalité, comme dans l'étude de Kocher et Chabert, il est apparu que les personnes suivies étaient isolées tout au long de leur vie. Bien qu'intégrées sur le plan professionnel, ces personnes étaient méfiantes, n'avaient peu ou pas de relations en dehors des contacts obligés ${ }^{7}$.

Il reste difficile de savoir si toutes les personnes avec ce profil psychologique présenteront un syndrome de Diogène, une fois à la retraite. De ce fait une intervention précoce paraît difficile, voire impossible ${ }^{8}$. Reste le consentement libre et éclairé, dans le respect de l'autonomie du malade ou «malade potentiel». Ceci est à prendre en compte de manière impérative.

De nombreux auteurs évoquent la fonction potentiellement protectrice de l'isolement «pathologique» tel qu'il peut être ressenti dans le syndrome de Diogène. Y. Kocher évoque une «angoisse de morcellement », qui induirait un comportement protecteur, justifiant d'amasser de façon compulsive et de vivre dans une sorte de réclusion volontaire et protectrice. Dans ce sens, la mise en évidence des facteurs ayant conduit à cet isolement.

Bien que bousculant le sens traditionnel des valeurs et les standards de vie habituels, les personnes présentant un syndrome de Diogène ne laissent personne indifférent. L'exaspération du voisinage et des autorités, le sentiment d'impuissance des intervenants, la difficulté des contacts favoriseraient avant réflexion une action, mais potentiellement dommageable pour la personne.

Nous tenons à privilégier le respect de la personne, l'action par petites touches, afin de gagner la confiance, afin d'éviter «l'éclatement» de la personne prise en soins. Toutes montrent des difficultés relationnelles qui sont de surcroît renforcées par les difficultés liées à l'âge, à la maladie, à la perte d'indépendance et d'autonomie.

Les intervenants sont alors souvent pris individuellement dans des conflits de valeurs qu'il importe de partager collégialement en équipe multidisciplinaire. Le regard des différents intervenants alors ne pouvant qu'enrichir le panel de solutions. Il ne faudra, en aucun cas, s'enfermer dans l'agir seul, avec pour risque de s'éloigner des valeurs éthiques et du respect du malade.

Peu à peu la satisfaction de découvrir des personnalités ô combien complexes, mais ô combien riches, nous motive dans la poursuite de la prise en charge, malgré les obstacles et les embûches.

De ce syndrome se dégage une souffrance fondamentalement humaine, à laquelle il s'agit d'apporter la réponse la plus adéquate possible.

\section{Notes}

1 D. MACMILLAN et P. SHAW, "Senile Breakdown in Standards of Personal and Environmental Cleanliness ", British Medical Journal, 1966, vol. 2, p. 1032-1037.

2 A.N.G. CLARK, G.D. MANKIKAR et I. GRAY, "Diogenes Syndrome A Clinical Study of Gross Neglect in Old Age », The Lancet, 1975, vol. 1, p. 366-368; N. BERLYNE, «Diogenes syndrome» [letter], The Lancet, 1975, vol. 1, p. 515 ; J. TWOMEY, «Diogenes syndrome » [letter], The Lancet, 1975, vol. 1, p. 515 ; A.N.G. CLARK, "The Diogenes syndrome», Nursing Times, 1975, p. $800-802$.

3 J. CALVO MELENDRO et al., "Sìndrome de Diògenes: Un cuadro clìnico frecuente en la vejez y poco conocido ", Actas dermoSifilographicas, 1976, vol. 67, p. 225-232; K. KAFETZ et J. COX, "Alcohol Excess and the Senile Squalor Syndrome », Journal of American Geriatric Society, 1982 , vol. 30, p. 706; J. KLOSTERKOTTER et U.H. PETERS, "Das Diogenes-Syndrom », Fortschr. Neurol. Psychiat., 1985, vol. 53, p. 427-434; W. CYBULSKA et J. RUCKINSKI, "Gross Self-Neglect in Old Age », Br. J. Hosp. Med., 1986, vol. 34, p. 21-25; T.S. RADEBAUGH, F.J. HOOPER et E.M GRUENBERG, "The Social Breakdown Syndrome in the Elderly Population Living in the Community: the Helping Study », $B r$. J. Psychiatry, 1987, vol. 151, p. 341-346; P. HETTIARATCHY et J. MANTHORPE, "The "Hidden" Nature or Self-Neglect», Care of the Elderly, 1989, vol. 1, no 1, p. 14-15; M.W. ORREL, B.J. SAHAKIAN et K. BERGMANN, "Self-Neglect and Frontal Lobe Dysfunction», Br. J. of Psychiatry, 1989, vol. 155, p. 101-105. P.G. REED et V.E. LEONARD, "An Analysis of the Concept of Self-Neglect», ANS Adv. Nurs. Sci., 1989, Oct, vol. 12 , no 1, p. 39-53; A. CACHIN, P. KUNULER et J. DEMAUREX, «Soubeyran foyer de jour pour personnes agées », Gérontologie, 1990, vol. 73, p. 15-24 ; G.S. UNGVARI et P.M. HANTZ, "Social Breakdown in the Elderly, I. Case studies and management", Comprehensive Psychiatry, 1991, vol. 32, no 5, p. 440-444; G.S. UNGVARI et P.M. HANTZ, "Social Breakdown in the Elderly, II. Sociodemographic data and psychopathology ", Comprehensive Psychiatry, 1991, vol. 32, no 5, p. 445-449; C. MARTIN-HUNYADI et M. BERTHEL, "Le syndrome de Diogène », Médecine et Hygiène, 1991, vol. 49, p. 1389-1393; P.B. ROBBEN, «Zelfverwaarlozing bij ouderen over thuislozen en het Diogenes-Syndroom », Tijdschr. Gerontol.
Geriatr., 1991, vol. 22, no 5, p. 167-171; M. GANNON et J. O'BOYLE, "Diogenes Syndrome », Ir. Med. J., 1992, vol. 85, no 4, p. 124 ; W. HOFFMANN, "Das DiogenesSyndrom : Leben zwischen allerlei Krimskrams", Geriatrie Praxis, 1992, vol. 2, p. 43-44; J.J. DE LA GANDARA MARTIN et T. ALVAREZ ALVAREZ-MONTESERIN, «Repercusiones psico- sociales de la soledad en las mujeres ancianas: sindrome de Diogenes ", Gereatrika, 1992, vol. 8, no 9, p. 433-438; A.J. COLE et T.P. GILETT, «A Case of Senile Self-Neglect in a Married Couple: Diogènes à Deux?", International Journal of Geriatric Psychiatry, 1992, vol. 7, p. 839-841; M.S. LACHS et al., "A Prospective Community-Based Pilot Study of Risk Factors for the Investigation of Elder Mistreatment», J. Am. Geriatr. Soc., 1994, Feb., vol. 42, no 2, p. 169-173 ; M.S. LACHS et K. PILLEMER, "Abuse and Neglect of Elderly Persons », NEJM, 1995, vol. 332, no 7, p. 437-443 ; C. COONEY et W. HAMID, "Review : Diogenes Syndrome», Age and Aging, 1995, p. 451-453; A. BAULON, "Éthique et personnes âgées », Gérontologie et société, no 80, mars 1997; E. HIRSCH, «Espace éthique: penser les valeurs de l'hôpital dans la cité», Gérontologie et société, no 80, mars 1997; G. HALLIDAY et al., "Community study of people who live in squalor», The Lancet, vol. 355, March 11, 2000, p. 882-886; O. FARALDI et al., "Syndrome de Diogène à ... deux », La revue de Gériatrie, Tome 25, vol. 7, sept. 2000, p. 517-520; A. BENNET et al., "Les amasseurs compulsifs ", Médecine et Hygiène, vol. 2318, le 18 octobre 2000, p. 2069-2074.

4 M.J. CHABERT et Y. KOCHER, "Le syndrome de Diogène », Soins Infirmiers, 1992, p. 9-15; M.J. CHABERT et Y. KOCHER, «Comment entrer en communication avec des personnes âgées qui nient leurs problèmes médicaux-sociaux", Médecine et Hygiène, 1992, vol. 50, p. 2990-2992; Y. KOCHER et M.J. CHABERT, "Le syndrome de Diogène ", Gérontologie et Société, 1993, vol. 64, p. 133-144.

5 DSM IIIR, Manuel de diagnostique et statistique des troubles mentaux, Éd. Masson, 1989.

6 D. O'MAHONY et J. GRIMLEY EVANS, «Diogenes Syndrome» [letter], Br. J. Psychiatry, 1994, vol. 164, p. 705-706.

7 R. MOORE, «Diogenes Syndrome», Nursing Times, 1989, vol. 85, no 30, p. 46-48.

8 A.N.G. CLARK, "Management of Severe Self-Neglect in Old Age», Care of the Elderly, 1989, vol. 1, no 1, p. 25-27; M.J. CHABERT et Y. KOCHER, «Le syndrome de Diogène", Soins Infirmier; F. LOEW et J.-J. NAEF, «De l'isolement social au syndrome de Diogène", Cahiers psychiatriques, Santé mentale en ville, numéro spécial en cours de publication. 